\title{
The Research on SME's Equity Value with Stochastic Pricing Method
}

\author{
Changbing Yang ${ }^{1,2,3}$ Mu Zhang ${ }^{3}$ \\ 1 Guizhou Institute for Urban Economics and Development, Guizhou University of Finance and \\ Economics, Guiyang Guizhou 550025, China \\ 2Guizhou Institution for Technology Innovation \& Entrepreneurship Investment, Guizhou \\ University of Finance and Economics, Guiyang Guizhou 550025, China \\ 3School of Finance, Guizhou University of Finance and Economics, Guiyang Guizhou 550025, \\ China
}

\section{科技型中小企业股权价值的随机定价方法研究}

\author{
杨昌兵 ${ }^{1,2,3}$ 张目 $^{3}$ \\ 1 贵州财经大学 贵州城镇经济与发展研究院, 贵州 贵阳 550025 \\ 2 贵州财经大学 贵州科技创新创业投资研究院, 贵州 贵阳 550025 \\ 3 贵州财经大学 金融学院, 贵州 贵阳 550025
}

\begin{abstract}
In order to value the equity of SME effectively, we modify the traditional DCF model, namely, we use stochastic variable to describe the factors which have direct impact on cash-in and cash-out; then we use stochastic equation to portray the change of equity valuation; finally, we will simulate the the change of equity valuation within some periods by Monte Carlo simulation. In accordance with the result coming from the simulation, we find that there exists a big probability that the company will bankrupt, however, their growth speed is extremely high and potential value is decent, which rightly fit the realistic situation. therefore, this modified method has ,to some extent, reference value in valuing the equity of SME.
\end{abstract}

Keywords: SME, equity value, stochastic process, Monte Carlo simulation

\section{摘要}

为有效的评估科技型中小企业的股权价值 （假设债权价值为 0 ）, 我们通过改进的贴 现现金流的方法, 即将导致现金流流入流出 的一些变量当中引入随机因素, 然后通过随 机方程式来描述企业股权价值的变动, 最后 利用 Monte Carlo 模拟来刻画公司股权价值 在一段时间内的变化情况。通过模拟我们发 现科技型中小企业相对成熟企业而言存在 巨大的破产风险, 但是其成长的速度非常 快, 而且潜在价值巨大, 这一模拟结果恰好 和我们现实情况比较吻合。因此, 这种方法 对于我们以后评估科技型中小企业的股权 价值具有很大的借鉴作用。

关键字: 科技型中小企业, 股权价值, 随机 过程, Monte Carlo 模拟

\section{1. 引言}

对于企业价值的评估最早可以追溯 到 1906 年, 当时 Irving Fisher 在他的《资本 与收入的性质》一书中提出资本的价值是收 入的折现。1938 年 Williams 在他的《投资 
价值理论》一书中对 Irving Fisher 的理论进 一步进行了研究, 提出公司的价值在于企业 在存续期内能够产生的所有现金流通过恰 当的折现率进行折现的现值, 这一想法构建 了贴现现金流估值的基本雉形。

1952 年 Sharp 提出资本资产定价模型 (CAPM) 为确定贴现现金流当中的贴现率 奠定了基础。

1962 年 Myron J.Gordon 更进一步的发 展出了零增长股利贴现模型, 固定增长股利 贴现模型等。

1964 年 Miller 和 Modigliani 提出 MM 定理, 认为企业的价值与公司的资本结构无 关, 与是否发放股利也无关。企业的价值取 决于公司未来的发展机会以及资本成本。因 此, 国外的学者渐渐将方向转向贴现现金 流, 权益现金流模型, 并且这种模型也渐渐 变得更加成熟。

随着资本市场的进一步发展, 1991 年 Stern Stewart 咨询公司将 EVA 的概念引入估 值领域。这种方法完全从经济学的视角出 发, 直接用营业的净利润减去权益和负债然 后得到企业的经济增加值, 作为企业价值的 来源, 然后再将其通过折现率进行折现, 这 样就能够得到企业的价值。

除了以上国外学者所做的贡献之外, 国 内的学者在这个领域也有不少研究。

王少豪（2002） ${ }^{[1]}$ 根据科技型中小企业 的特点, 分别分析了现金流贴现法, 重置成 本法以及我们没有涉及的期权法在我国的 应用。石晓军 (2003) ${ }^{[2]}$ 通过根据资本资产 定价模型和贴现现金流模型, 对非上市公司 的企业价值评估进行了研究。曾繁荣 (2009) ${ }^{[3]}$ 在自由现金流的基础上对美的集团收购 小天鹅的案例进行了分析。

本篇论文着重的考虑了现金流来源的 分析与预测, 并且与传统的现金流来源分析 不同，在充分考虑了科技型中小企业自身特 点的情形下, 我们通过预测企业未来客户的 价值和数目从来间接的评估企业的股权价 值。模型将建立在随机的分析框架之下, 即, 假设影响企业现金流的一些重要变量是具 有某种特性随机过程, 在随机的框架下分析 这类企业的股权价值存在两个方面的优势:
一方面科技型中小企业其本身在发展的初 始过程当中就具有很大的不确定性, 所以利 用随机过程 (主要是维纳过程) 去刻画影响 企业现金流的一些主要变量, 充分符合企业 的特性; 另一方面, 随机过程刻画的精确程 度相对传统的贴现现金流而言更高。

\section{2. 模型建立}

\section{1 收入模型}

根据 $\operatorname{Bass}(1969)^{[4]}$ 我们将消费者分为两 类: 一类是 “创新者” , 这类消费者敢于尝 试新颖的产品, 换句话说, 也就是企业新产 品能够获得的第一批客户。这类消费者主要 是受外部因素影响, 例如媒体, 政府政策等; 另一类是 “模仿者”, 这类消费者不敢尝试 最新的产品, 但是他们的消费行为容易受到 别人影响, 即喜欢模仿 “创新者” 的消费行 为。所以, 消费企业产品或者服务的 “模仿 者” 数目取决于 “创新者” 数目。更进一步, 我们假设 “创新者” 数目需要达到一个最小 值 $N^{\mathrm{min}}$, 当且仅当 “创新者” 数目达到 $N^{\mathrm{min}}$ 才能够吸引来足够多的 “模仿者” 并且使得 模仿者的数目能够自行扩散, 而这个最小数 目 $N^{\min }$ 我们也称作 “临界数目”。

在自行扩散过程当中, 能够获得的最大 消费者数目为 $N_{t}^{\max }$, 其表达式如下:

$$
N_{t}^{\max }=m_{t} F N
$$

其中 $m_{t}$ 表示企业在时间 $\mathrm{t}$ 的时候在市场当中 的潜在份额。但是, 根据正反馈效应, 在给 定的时间当中，如果企业没有获得 “临界数 目” 的消费者数量, 从而使得消费者数目能 够自行扩散, 那么, 从长期而言, 消费企业 产品的消费者数量将不断减少。所以, 科技 型中小企业将面临两种情况: 第一种, 企业 能够获得 “临界数目” 的消费者数量, 从而 使得企业能够继续存活; 第二种, 企业无法 获得 “临界数目” 的消费者数目, 从而使得 企业消费者数目不断的减少。

假设企业的产品在市场上发行时间为 $\Delta t$ 之后, 所能够吸引来的 “创新者” 数目为 $\Delta N_{t}$ 并且 $\Delta N_{t}$ 服从泊松分布, 其表达式如下:

$\Delta N_{t} \sim P\left(\lambda_{t} \Delta t\right)$ 
其中 $\lambda_{t}$ 平均到达率。

根据泊松分布的收敛性我们知道, 泊松分 布会向正态分布收敛。在连续时间和状态空 间下, 我们用下面的随机微分方程来描述客 户数目的动态变化, 并且通过这个随机微分 方程将两种分布结合起来:

$$
d N_{t}=\lambda_{t} d t+\sqrt{\lambda_{t}} d W_{t}^{N}
$$

其中 $W_{t}^{N}$ 是一个标准维纳过程。

假设企业按照一定的比率丧失已获得的 客户，我们用 $\xi$ 表示这一比率，即丧失的客 户的数目可以表示为 $\xi N_{t}$ 。按照这种思路, 我们可以推导出客户数目在没有达到 “临界

值”之前的动态变化情况，其公式下：

$$
d N_{t}=\left(\lambda_{t}-\xi N_{t}\right) d t+\sqrt{\lambda_{t}} d W_{t}^{N}
$$

其中 $\lambda_{t}$ 是关于公司营销策略或者市场竞 争程度等的随机函数。此处, 我们假设 $\lambda_{t}$ 服 从伊藤过程, 其表达式如下:

$$
\frac{d \lambda_{t}}{\lambda_{t}}=\alpha_{t} d t+\delta d W_{t}^{\lambda}
$$

其中, $\alpha_{t}$ 表示期望变动率, $\delta$ 表示 $\lambda_{t}$ 变动 率的波动率。同时, $\alpha_{t}$ 是上面提到的两个因 素的函数, 在长期而言, 由于 “创新者” 数 目是不断减少的, 所以我们认为从长期的角 度看 $\alpha_{t}$ 是一个负值。由于长期来说, 原本具 有创新性的产品或者服务会变得渐渐过时, 同时，新的竞争者会不断的进入市场当中， 所以，我们认为上面假定的 “创新者” 数目 在长期而言是不断减少的, 这是一个合理的 假设。

当“创新者”数目达到或者超过“临界值”, 即 $N_{t} \geq N^{\mathrm{min}}$ ， “模仿者” 数目就会进行自我 扩散直到达到最大可获得的消费者数目, $N^{\max }$ 。根据 Mansfiled(1961) ${ }^{[5]}$ 内部影响模 型, 即扩散是根据社会系统中成员之间的相 互交流。所以，当 “创新者” 数目达到或者 超过 “临界值” ，即 $N_{t} \geq N^{\mathrm{min}}$ 时, 消费者数 目的变化情况如下面的公式所示:

$$
d N_{t}=N_{t} \frac{q}{N^{\max }}\left(N^{\max }-N_{t}\right) d t
$$

其中 $q$ 表示内部影响的相关系数, 反应了 前面的消费者 $N_{t}$ 和后面的消费者 $N^{\max }-N_{t}$ 之间的相互作用。出于简便性的考虑, 我们 用下面的公式来化简（6）式的部分内容:

$$
\omega_{t}=\frac{q}{N_{t}^{\max }}\left(N_{t}^{\max }-N_{t}\right)
$$

如果我们再将客户损失考虑进去, 那么, 最终我们会得到如下的公式:

$$
d N_{t}=\left(\omega_{t}-\xi\right) N_{t} d t
$$

我们用 $t^{f}$ 表示达到“临界值”的初始时刻, 其公式表示如下:

$$
t^{f}=\sup \left(t: N_{t} \leq N_{t}^{\max }\right)
$$

利用公式（4）（8）（9）我们能得到下 面描述客户数目变化的一般公式:

$d N_{t}= \begin{cases}\left(\lambda_{t}-\xi N_{t}\right) d t+\sqrt{\lambda_{t}} d W_{t}^{N} & t<t^{f} \\ \left(\omega_{t}-\xi\right) N_{t} d t & t \geq t^{f}\end{cases}$

由于竞争者不断的进入市场, 所以我们假 设企业在时间 $\mathrm{t}$ 时刻能够获得的最大的市场 份额, $m_{t}$, 在企业获得 “临界值” 数目的消 费者之前是不断下降的 ${ }^{[7]}$; 当消费者数目达 到或者超过 “临界值” 那么企业所能够获得 最大的市场份额保持不变。根据上述描述, 我们能够获得下公式:

$$
\frac{d m_{t}}{m_{t}}= \begin{cases}-\beta d t & t<t^{f} \\ 0 & t \geq t^{f}\end{cases}
$$

其中 $\beta$ 表示 $m_{t}$ 的下降速率，表示了整个 市场的竞争激烈程度。 $\beta$ 越大表示市场竞争 的激烈程度越大, 同时表示企业需要获得 “临界值” 数目的消费者所需要花费的时间 越长, 能够获得市场份额越小。

企业的总收入， $R_{t}$, 等于企业获得的消费 者人数乘以每一个消费者能够给企业带来 的平均收入, $p_{t}$ :

$$
R_{t}=p_{t} N_{t}
$$

每个消费者给企业带来的平均收入, $p_{t}$, 是不确定的, 我们假定它服从伊藤过程, 其 表达式如下:

$$
\frac{d p_{t}}{p_{t}}=\mu_{t} d t+\sigma d W_{t}^{p}
$$

其中 $\mu_{t}$ 表示平均收入的期望增长率, $\sigma$ 表 示平均收入的增长率的波动率。我们假设期 
Risk Analysis and Crisis Response in Big Data Era (RAC-16)

望增长率 $\mu_{t}$ 随着时间的不断推移可以不断 的进行调整, 但是, 当企业成熟的时候, $\mu_{t}$ 确定性的趋向于一个正数, $\mu^{*}$, 我们用下面

的公式进行表述:

$d \mu_{t}=\kappa_{\mu}\left(\mu *-\mu_{t}\right) d t$

其中 $\kappa_{\mu}$ 表示调整的速度。

\section{2 成本模型}

假设公司的成本函数, $C_{t}$, 包括三个部分: (1) 固定成本 $F ;$ （2）变动成本, 假设变 动成本和收入成比例, 其比例系数为 $\gamma_{t} ;$ （3)

获得新顾客所需要的成本 $C_{t}^{A}$ 。这样我们就 可以将成本函数写成下面的形式:

$$
C_{t}=F+\gamma_{t} R_{t}+C_{t}^{A}
$$

假设获得消费者所需要的成本, $C_{t}^{A}$, 等于 平均获得每个消费者所需要的成本, $c^{A}$, 乘 以所有在时刻 $t$ 时消费者的总数量，式如下： $C_{t}^{A}=c^{A}\left(d N_{t}+\xi N_{t} d t\right) \frac{1}{d t}$

假设变动成本与收入之间的比例系数, $\gamma_{t}$, 在长期而言随机的趋向于平均 $\gamma^{*}$, 如下:

$d \gamma_{t}=\kappa_{\gamma}\left(\gamma^{*}-\gamma\right) d t+\varphi d W_{t}^{\gamma}$

其中, $\varphi$, 表示比例系数的波动率; $\boldsymbol{\kappa}_{\gamma}$ 表示均值回归速度参数。

\section{3 企业破产情形}

假设公司的所有固定资产为 $T P$, 其平均折 旧率为 $\theta$; 息税前收入为 $E B I T_{t}$, 所以 $E B I T_{t}$ 的表达式如下:

$$
E B I T_{t}=R_{t}-C_{t}-\theta T P_{t}
$$

我们假设公司税率为 $\varepsilon_{c}$, 这样企业的税后收 入, $Y_{t}$, 可以表示为:

$$
Y_{t}=\left(1-\varepsilon_{c}\right) E B I T_{t}
$$

假设企业对于资本的投资占收入的比例是 固定的, $\beta$ 。所以, 企业的累积资本就完全
取决于两个部分, 一部分是折旧 $\theta T P_{t}$, 另一部 分则是投资 $\beta R_{t}$, 所以企业总资本或者累计 资本就可以表示为:

$$
d T P_{t}=\left(-\theta T P_{t}+\beta R_{t}\right) d t
$$

假设企业的现金余额为 $X_{t}$, 并且按照固定 的无风险利率 $r$ 赚取利息, 当然这边我们也 可以假设 $r$ 是一个随机过程, 但是为了便于 分析我们不做那样的假设。这样积累的现金 流的总量就可以表示成如下公式: $d X_{t}=\left(r X_{t}+Y_{t}+\theta T P-\beta R_{t}\right)$

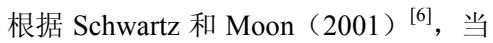
企业现金余额低于一个负数 $X^{*}$ 时, 企业将 宣布破产, 而且 $X^{*}$ 是提前被设定的且是宏 观因素, 公司现有资产和未来发展潜力等的 函数。

\section{4 企业价值}

因为我们评估的主要是科技型中小企 业在成熟之前这段时间的价值, 所以, 我们 假设这个时间段为 $T_{h}$, 并且通过将未来这段 时间的期望值进行贴现, 然后, 我们得到企 业股权现值。企业的价值主要包括两个部 分: 一部分是 $X_{T_{h}}$; 另一部分是 $R_{T_{h}}-C_{T_{h}}$ 。

假设整个市场是风险中性的, 并且为了便于 分析, 我们利用无风险利率对上面两个部分 进行贴现。所以, 公司在时间 $t$ 时刻的价值 为:

$V_{t}=E\left[X_{T_{h}}+\max \left\{\left(R_{T_{h}}-C_{T_{h}}\right), 0\right\} \mid F_{t}\right] e^{-r\left(T_{h}-t\right)}$

其中 $F_{t}$ 表示在时间 $t$ 的时刻所有信息所 组成的集合。

通过以上的分析, 我们可以把企业股权价值 函数写成以下的方式:

$$
V_{t}=V\left(N_{t}, \lambda_{t}, m_{t}, p_{t}, \gamma_{t}, X_{t}, T P_{t}, t\right)
$$

对等式 (29) 应用伊藤引理, 我们可以得到 以下关于公司价值的偏微分方程:

$d V_{t}=\frac{\partial V_{t}}{\partial N_{t}} d N_{t}+\frac{\partial V_{t}}{\partial \lambda_{t}} d \lambda_{t}+\frac{\partial V_{t}}{\partial m_{t}} d m_{t}+\frac{\partial V t}{\partial p_{t}} d p_{t}+$

$\frac{\partial V_{t}}{\partial \gamma_{t}} d \gamma_{t}+\frac{\partial V_{t}}{\partial X_{t}} d X_{t}+\frac{\partial V_{t}}{\partial T P_{t}} d T P_{t}+\frac{\partial V_{t}}{\partial t} d t+$

$\frac{1}{2} \frac{\partial^{2} V_{t}}{\partial N_{t}^{2}} d N_{t}^{2}+\frac{1}{2} \frac{\partial^{2} V_{t}}{\partial \lambda_{t}^{2}} d \lambda_{t}^{2}+\frac{1}{2} \frac{\partial^{2} V_{t}}{\partial p_{t}^{2}} d p_{t}^{2}+$ 
Risk Analysis and Crisis Response in Big Data Era (RAC-16)

$$
\begin{aligned}
& \frac{1}{2} \frac{\partial^{2} V_{t}}{\partial \gamma_{t}^{2}} d \gamma_{t}^{2}+\frac{\partial V_{t}}{\partial N_{t}} \frac{\partial V_{t}}{\partial \lambda_{t}} d N_{t} d \lambda_{t}+ \\
& \frac{\partial V_{t}}{\partial N_{t}} \frac{\partial V_{t}}{\partial p_{t}} d N_{t} d p_{t}+\frac{\partial V_{t}}{\partial N_{t}} \frac{\partial V_{t}}{\partial \gamma_{t}} d N_{t} d \gamma_{t} \\
& +\frac{\partial V_{t}}{\partial \lambda_{t}} \frac{\partial V_{t}}{\partial p_{t}} d \lambda_{t} d p_{t}+\frac{\partial V_{t}}{\partial \lambda_{t}} \frac{\partial V_{t}}{\partial \gamma_{t}} d \lambda_{t} d \gamma_{t} \\
& +\frac{\partial V_{t}}{\partial p_{t}} \frac{\partial V_{t}}{\gamma_{t}} d p_{t} d \gamma_{t}
\end{aligned}
$$

在风险中性的条件下, 计算 $d V_{t}$ 的期望 值我们就能够获得企业价值的基本偏微分 方程, 当然这个方程存在着很多边界条件。 根据上述模型我们知道, 企业的破产条件存 在路径依赖, 所以该偏微分方程的解不存 在。但是, 我们可以对上述模型采用离散时 间模型进行近似, 然后, 依靠 Monte Carlo 模拟获得企业价值。由于篇幅有限我们将直 接给出模拟的结果而省略相关的公式推导 过程。

\section{Monte Carlo 模拟}

为了更好的解释我们的模型，我们采用 Monte Carlo 方法来模拟一个虚构的科技型 中小企业股权价值的演变情况。我们考虑的 企业是该企业还没有取得 “临界值” 客户数 目, 并且 $N_{0}=5000 ; N^{\max }=25000$ 。假设所有 随机过程之间不相关, 未来不存在融资的可 能性。当公司的现金余额小于 0 的时候，即 认为公司的价值为 0 。出于方便起见, 我们 假设 $\lambda_{t}$ 的预期变化率 $\alpha_{t}$ 是负的常数, 价值跨 度为 20 年, 同时 $\mathrm{V} t$ 以季度为单位, 一共运 行的次数为 50000 。图 1 是我们根据模拟得 出来的价值和频率关系图, 其中横轴表示企 业价值, 纵轴表示达到这些价值的频率。

根据我们选定的参数, 我们能得到在 $t_{0}$ 时刻, 企业预期的股权价值为 $24.4 \mathrm{mn}$, 且 小部分时候股权价值甚至能超过 $150 \mathrm{mn}$, 但 是通过模拟出来的图形我们发现至少有 $18 \%$ 的概率企业会破产，即股权价值为 0 。 除此以外, 有 $19 \%$ 左右的概率企业的股权价 值在 1-5mn 之间徘徊, 尽管企业不会破产, 但是这只是预期股权价值当中很小的一部 分。

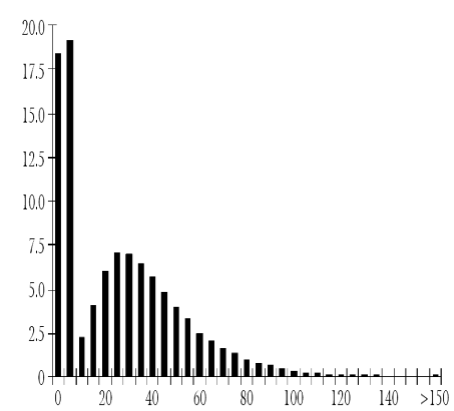

图 1 股权价值与频率

注：横轴表示股权价值, 单位为百万 $(\mathrm{mn})$ ； 纵轴表示达到某些股权价值的频率

\section{4. 结论}

根据上面 Monte Carlo 模拟结果我们发 现，科技型中小企业存在巨大的破产可能 性, 但是它们的增长速度和潜在价值也十分 可观, 这一模拟结果与我们所了解的现实情 况也是极其符合。所以, 我们上面提出这种 对于科技型中小企业股权价值的评估方法 存在一定的现实可靠性。

\section{致谢}

本文为国家自然科学基金地区项目《贷款风 险补偿资金对科技型中小企业信贷配给的 影响机理研究》(71263011)的阶段性成果之

\section{参考文献}

[1]王少豪.企业并购中协同效应与控股权价 值的评估.中国资产评估，2002，4(4): 18-20.

[2]石晓军. 非上市公司价值评价: 基于 CAPM 的 DCF 方法与实证. 商业研究, 2003, (3): 72-74.

［3］曾繁荣; 王志仁. 我国企业并购的风险与 控制分析.中国管理信息化. Vol.12 No.28,2019.5

[4] Bass Frank M (1969), "A New-Product Growth Model for Consumer Durables", 
Risk Analysis and Crisis Response in Big Data Era (RAC-16)

Management Science, Vol. 15,pp. 215-227.

[5] Schwartz Eduardo S and Moon Mark (2001), "Rational Pricing of Internet Companies Revisited", The Financial Review, Vol. 36, pp. 7-26

[6] Mansfield Edwin (1961),"Technological Change and the Rate of Imitation",
Econometrica, Vol. 29,pp. 741-766.

[7] M. Zhang, Z.F. Zhou. A Credit rating model for enterprises based on projection pursuit and k-means clustering algorithm. Journal of Risk Analysis and Crisis Response, 2012, 2(2): 131-138. 